\title{
O rastro da COVID-19 no mundo: Reflexão teórica sobre a repercussão da pandemia e seus desdobramentos na saúde dos profissionais de enfermagem
}

\author{
The track of COVID-19 in the world: theoretical reflection on the repercussion of the pandemic \\ and its consequences on the health of nursing professionals
}

La ruta del COVID-19 en el mundo: Reflexión teórica sobre las repercusiones de la pandemia y sus consecuencias en la salud de los profesionales de enfermeira

Recebido: 12/02/2021 | Revisado: 18/02/2021 | Aceito: 25/02/2021 | Publicado: 03/03/2021

\author{
Maria Lucia Costa de Moura \\ ORCID: https://orcid.org/0000-0002-0700-9564 \\ Universidade Paulista, Brasil \\ E-mail: lucidalv@yahoo.com.br \\ Suely Lopes de Azevedo \\ ORCID: https://orcid.org/0000-0003-1107-3427 \\ Universidade Federal Fluminense, Brasil \\ E-mail: suelyazevedo@id.uff.br \\ Juliana da Silva Parente \\ ORCID: https://orcid.org/0000-0003-1019-8025 \\ Universidade Federal Fluminense, Brasil \\ E-mail: julianaparente@id.uff.br \\ Aline Silva da Fonte Santa Rosa de Oliveira \\ ORCID: https://orcid.org/0000-0002-4070-7436 \\ Faculdade Bezerra de Araújo, Brasil \\ E-mail:alinefonte@globo.com \\ Flavia Carine Barreto Brandão \\ ORCID: https://orcid.org/0000-0001-8252-0365 \\ Hospital do Subúrbio-SSA, Brasil \\ E-mail: Flaviabrandao_@hotmail.com
}

\begin{abstract}
Resumo
Objetivo: Refletir o impacto da pandemia da COVID-19 nos profissionais que atuam diretamente nos serviços de saúde junto aos pacientes acometidos pela doença. Método: Ensaio teórico-reflexivo, descritivo, qualitativo produzido a partir da análise crítica de diferentes bases de dados, manuais, sites institucionais e jornalísticos nacionais e internacionais em busca de dados atualizados para a contextualização da temática. Resultados e Discussão: Ressalta-se que, seja no serviço administrativo ao assistencial, todas as organizações de saúde, nacionais e internacionais, estão empenhadas em não medir esforços na implementação de protocolos e medidas de proteção e prevenção da doença, bem como, proteger os profissionais de saúde que estão trabalhando diretamente no enfrentamento da COVID-19. Nesta perspectiva, as autoras buscam contribuir com os serviços de saúde, no sentido de despertar para ações que visam a segurança do paciente e dos profissionais envolvidos no cuidado. Conclusão: a reflexão do estudo remete para o grande desafio da humanidade na busca por uma solução que garanta uma assistência digna e segura para enfrentar os desdobramentos da pandemia. É evidente que o desafio vai além do controle da doença, tornando visível a precariedade dos cenários e dos sistemas de saúde ao redor do mundo com desigualdade social, dificuldade de acesso aos serviços públicos, déficit de profissionais, processos de gestão em saúde inoperantes, falta de recursos humanos e materiais que vão desde o fornecimento de EPI até qualificação em serviço. Esses fatores influenciam no aumento do número de óbitos e corroboram para maior vulnerabilidade dos profissionais de saúde.
\end{abstract}

Palavras-chave: COVID-19; Profissionais de saúde; Pandemia; Enfermagem; Coronavírus.

\begin{abstract}
Objective: To reflect the impact of the COVID-19 pandemic on professionals who work directly in health services with patients affected by the disease. Method: Theoretical-reflective, descriptive, qualitative essay produced from the critical analysis of different databases, manuals, institutional and national and international journalistic sites in search of updated data to contextualize the theme. Results and Discussion: It is noteworthy that, whether in the administrative service to assistance, all health organizations, national and international, are committed to spare no effort in the implementation of protocols and measures for the protection and prevention of disease, as well as protecting the health professionals who are working directly to face COVID-19. In this perspective, the authors seek to contribute to health services, in the sense of awakening to actions aimed at the safety of patients and professionals involved in care.
\end{abstract}


Conclusion: the study's reflection refers to humanity's great challenge in the search for a solution that guarantees a dignified and safe assistance to face the consequences of the pandemic. It is evident that the challenge goes beyond the control of the disease, making visible the precariousness of health scenarios and systems around the world with social inequality, difficulty in accessing public services, deficit of professionals, inoperative health management processes, lack of human and material resources ranging from the provision of PPE to qualification in service. These factors influence the increase in the number of deaths and corroborate the greater vulnerability of health professionals.

Keywords: COVID-19; Health professionals; Pandemic; Nursing.

\section{Resumen}

Objetivo: Reflejar el impacto de la pandemia COVID-19 en los profesionales que trabajan directamente en los servicios de salud con pacientes afectados por la enfermedad. Método: Ensayo teórico-reflexivo, descriptivo, cualitativo elaborado a partir del análisis crítico de diferentes bases de datos, manuales, sitios periodísticos institucionales y nacionales e internacionales en búsqueda de datos actualizados para contextualizar el tema. Resultados y Discusión: Es de destacar que, ya sea en el servicio administrativo a la asistencia, todas las organizaciones de salud, nacionales e internacionales, están comprometidas a no escatimar esfuerzos en la implementación de protocolos y medidas de protección y prevención de enfermedades, así como a proteger los profesionales de la salud que están trabajando directamente para enfrentar el COVID-19. En esta perspectiva, los autores buscan contribuir a los servicios de salud, en el sentido de despertar a acciones dirigidas a la seguridad de los pacientes y profesionales involucrados en el cuidado. Conclusión: la reflexión del estudio se refiere al gran desafío de la humanidad en la búsqueda de una solución que garantice una asistencia digna y segura para enfrentar las consecuencias de la pandemia. Es evidente que el desafío va más allá del control de la enfermedad, visibilizando la precariedad de los escenarios y sistemas de salud alrededor del mundo con desigualdad social, dificultad para acceder a los servicios públicos, déficit de profesionales, procesos de gestión de la salud inoperantes, falta de recursos humanos y materiales. recursos que van desde la provisión de EPI hasta la calificación en servicio. Estos factores influyen en el aumento del número de muertes y corroboran la mayor vulnerabilidad de los profesionales de la salud.

Palabras clave: COVID-19; Profesionales de la salud; Pandemia; Enfermería.

\section{Introdução}

A década de 2020 ficou marcada pela identificação de um novo tipo de coronavírus, descoberto em 2019, onde o crescimento exponencial de casos graves de pneumonia infecciosa, síndrome respiratória aguda grave, que afetou pessoas de todas as nações, raças e grupos socioeconômicos, evoluindo para um surto sem precedentes de proporções inimagináveis. Essa doença causou uma crise devastadora, inicialmente na China, o que fez com que a Organização Mundial de Saúde (OMS) fizesse um alerta sobre a doença em 31 de dezembro de 2019 (OPAS, 2020; Shanafelt et al., 2020; WHO, 2020a).

Tal fato, conduziu o diretor da OMS (2020), em 30 de janeiro de 2020, em Genebra, a declarar que o surto global do novo coronavírus era uma Emergência de Saúde Pública de Importância Internacional (ESPII), enfatizando que o vírus tinha grande potencial para ser disseminado rapidamente, com proporções alarmantes e maior impacto nos países que possuíam sistemas de saúde mais frágeis e sem estrutura para controlar sua transmissão através de medidas eficazes de contenção, controle, diagnóstico e tratamento. Em 11 de março de 2020, a OMS reconheceu a pandemia da COVID-19, sendo um dos maiores desafios para a saúde pública no mundo devido à ameaça da doença e aumento do número de mortes (WHO, 2020b).

Ressalta-se que compreender a transmissão global do vírus em evolução é profundamente importante para uma resposta rápida e eficaz no âmbito da saúde pública, até porque pode-se prever surtos futuros. Nesse sentido, ficou evidente que o mundo precisava triplicar esforços para implementar políticas públicas eficazes, apoiar as pesquisas nas instituições nacionais e internacionais, para que os recursos humanos, materiais e financeiros possam criar medidas que garantem a transparência da situação da doença, seu avanço, tratamento e controle. Sabe-se que os casos confirmados da COVID-19, são largamente considerados uma subconta do acumulado real, e por isso governos Estaduais e Municipais começaram a diferenciar casos e mortes viáveis (Oliveira et al, 2020).

Observa-se que a pandemia do coronavírus, varreu o mundo e fez mais do que ceifar vidas e meios de subsistência da população mundial. Segundo a OMS (2020), a detecção e a propagação de um patógeno respiratório emergente são acompanhadas pela incerteza sobre as características epidemiológicas, clínicas e virais do novo vírus e particularmente sua 
habilidade de se espalhar na população humana e sua virulência, diante disso, a pandemia decorrente da infecção humana pelo novo coronavírus tem causado impactos com prejuízos globais de ordem política, social e econômica, tornando-se o maior desafio da saúde pública mundial.

O contexto é parecido ao que já aconteceu em outros momentos de surtos e doenças da população, quando houve disseminação de alguns vírus e bactérias que causaram alta taxa de morbidade e mortalidade na humanidade. Como sabemos pela história natural desta doença, as pessoas infectadas podem apresentar reações diversas como, por exemplo: não apresentarem sintomas (assintomáticas); terem sintomas leves que melhoram sem intervenção médica; sintomas moderados que necessitem de internação hospitalar, ou sintomas graves com necessidade de internação em unidades de terapia intensiva devido às complicações, o que pode levar à óbito (Velavan \& Meyer, 2020).

Corrobora-se com Jacob et al (2020), quando afirma que perante a inevitabilidade de emergência pública, fez-se necessário a reorganização das instituições de saúde, através de um processo de planejamento organizativo e estrutural com maior resolutividade no processo de cuidar dos pacientes com a COVID-19, com criação de protocolos clínicos de tratamento de atendimento para cada fase da doença, organização dos fluxos de leitos destinados para pacientes contaminados, testagem em massa, definição de áreas de isolamento social para maior controle e prevenção da saúde da população, além de medidas de biossegurança com fornecimento de equipamento de proteção individual para todos os profissionais de saúde.

Outro aspecto importante foi a rápida disseminação da COVID-19 no mundo, representada por alta taxas de contágio e mortalidade com maior gravidade dos sintomas, o que sobrecarregou fortemente os limites dos sistemas de saúde e colocou em risco a saúde física e mental dos profissionais de saúde, evidenciada pelo aumento do número de mortes destes que estavam atendendo na linha de frente. O receio em lidar com uma doença desconhecida, maior vulnerabilidade para se contaminar levou muitos trabalhadores ao isolamento de seus familiares, o que comprometeu toda a dinâmica dos serviços de saúde, representada pela crescente carga de trabalho, realocação do profissional fora da sua área de especialização, falta de capacitação, superlotação das unidades de saúde e ausência ou dificuldade na infraestrutura para o isolamento e tratamento adequado dos doentes além da falta dos materiais de biossegurança (Lai et al., 2019).

Diante do exposto, fica evidente que dentre os grupos de trabalhadores atingidos pela COVID-19, os profissionais de saúde têm maior risco de contaminação do que a população em geral, especialmente, aqueles com alta exposição, que prestam cuidado à população em diferentes contextos nos serviços de saúde. Em relação à Enfermagem, categoria que representa o maior número de profissionais da saúde, a situação se agrava, devido à baixa remuneração, desigualdades sociais, acúmulo de funções administrativas, falta de funcionários, prática das atividades assistenciais em situações precárias, sobrecarga dos horários de trabalho e acúmulo de vínculos empregatícios. Todos estes fatores foram agravados com a pandemia, o que contribuiu para o aumento do risco de infecção, caso se exponham ao vírus. É emergencial ações mais conscientes visando a saúde e o bem estar desses profissionais, principalmente, aqueles que trabalham diretamente no diagnóstico, tratamento e cuidado de pacientes infectados pelo novo coronavírus.

O estudo em tela tem como objetivo: refletir o impacto da pandemia nos profissionais de enfermagem que atuam diretamente junto aos pacientes acometidos pela COVID-19 nos serviços de saúde.

\section{Metodologia}

Trata-se de um ensaio-teórico reflexivo, com abordagem qualitativa. Como preconiza Pereira, et al., (2018) este tipo de estudo pode trazer uma riqueza de dados e informações de modo a contribuir com o saber na área de conhecimentos na qual for utilizada. Dessa forma, o ensaio teórico reflexivo tem como fundamentos a exposição lógica e reflexiva, além da argumentação minuciosa, com elevado grau de interpretação e julgamento pessoal. Consiste na exposição das ideias e pontos de vista do autor sobre determinado tema, buscando originalidade no enfoque, sem, contudo, explorar o tema de forma exaustiva (Severino, 2017). 
Assim, tal reflexão, constituída à luz da literatura, através do pensamento crítico dos seus autores, foi alicerçada na literatura nacional e internacional acerca da temática. Foi realizada uma busca de referências bibliográficas, no período dezembro de 2020 até a data de 09 fevereiro de 2021, com as palavras chaves: COVID-19; Profissionais de saúde; Pandemia; Enfermagem e Coronavírus nas bases de dados: PubMed/Medline, Scopus, Web of Science, SciELO e sites governamentais de instituições de saúde, além de documentos eletrônicos oficiais, jornais, protocolos, manuais e notas técnicas dos diferentes países, nos idiomas inglês, espanhol e português, com o propósito de identificar fontes relevantes e atualizadas sobre os casos da COVID-19 no mundo e seu impacto na saúde dos profissionais de saúde que atuaram na linha de frente do cuidado. Todo o material foi avaliado pelos autores e utilizado as informações relevantes na apresentação dos resultados e discussão abaixo.

\section{Resultados e Discussão}

Após leitura atenta do material selecionado, foi possível identificar quatro categorias temáticas: Nomeando a doença e decretando a pandemia, Impacto irreversível da pandemia nos profissionais de saúde: Mortes por COVID-19, A ciência à serviço da humanidade: a busca pela vacina e Imunização dos profissionais de saúde, apresentadas e discutidas a seguir, no intuito de propor reflexões acerca da temática em questão. Para isto, os autores apresentam evidências e dados relevantes sobre o tema.

\subsection{Nomeando a doença e decretando a pandemia}

Coronavírus é uma família de vírus que causam infecções respiratórias. Recebe esse nome porque tem aparência de coroa (corona, em espanhol). Esse agente infeccioso foi identificado pela primeira vez em humanos e isolado em 1937. Porém, só foi descrito como Coronavírus em 1965, quando a análise de perfil na microscopia revelou sua aparência. O novo coronavírus denominado SARS-CoV-2, foi divulgado pelo escritório da OMS na China, em 31 de dezembro de 2019, que informou casos de pneumonia de etiologia desconhecida detectados na cidade de Wuhan, província de Hubei. De acordo com relatos da mídia, o mercado em questão foi fechado em 1 de janeiro de 2020 para saneamento e desinfecção ambiental. Em 3 de janeiro de 2020 foi notificado pelas autoridades nacionais na China que na República Popular em Wuhan, tinha registro de 44 pacientes com pneumonia viral, sendo que 11 pessoas em estado grave, o que atraiu a atenção das autoridades de saúde no mundo (Lima, 2020). Em 9 de janeiro, após investigações das autoridades chinesas, a OMS (2020) informou que os casos de pneumonia relatados em Wuhan se tratavam de um novo vírus, da família coronavírus, que causa doenças que variam de um resfriado comum a doenças mais graves, como a Síndrome Respiratória do Oriente Médio (MERS) e a Síndrome Respiratória Aguda Grave (SARS).

Desde o dia 11 de março de 2020, a OMS passou a classificar oficialmente a infecção causada pelo novo Coronavírus, como pandemia. Num reconhecimento de que sua transmissão recorrente estava ocorrendo em diferentes partes do mundo e de forma simultânea, indicando que governos devem trabalhar para identificar novos casos e isolar pessoas através de uma política de saúde pública, voltada para evitar mortes e não apenas a proliferação da doença, principalmente, nos países mais pobres, populosos e vulneráveis (Greshko, 2021).

Desde então, surgiram estudos e polêmicas sobre o que provocou a pandemia no mundo. As primeiras infecções confirmadas na Europa e nos Estados Unidos, descobertas em janeiro, não provocaram as epidemias que se seguiram, de acordo com uma análise cuidadosa de centenas de genomas virais. A linha do tempo revisada pode esclarecer ambiguidades incômodas sobre a chegada da pandemia, estatísticos e especialistas em saúde pública dizem que não há evidência de que o coronavírus foi fabricado na China, e que o número de mortes é provavelmente muito maior do que o divulgado nos canais de comunicação oficial da Organização das Nações Unidas (ONU). A teoria sobre o vírus ter sido encontrado na natureza, levado para um laboratório para ser manipulado de forma artificial e deliberadamente ou acidentalmente ser liberado para a comunidade não são fundamentadas nas provas científicas (Astor et al., 2020). 
Em entrevista dada à revista National Geographic, o diretor do Instituto Nacional de Alergias e Doenças Infecciosas dos EUA, Anthony Tony Faci, acalmou o debate referindo, que a ciência comprova que se olharmos para a evolução do vírus nos morcegos e para a variante que circula agora, fica evidente a forma como as mutações evoluíram naturalmente. Estudos de vários biólogos evolucionistas confirmaram que houve evolução gradual do vírus, o que indica que ele evoluiu na natureza e depois saltou entre espécies ao longo dos anos, por isso, também, não acredita na possibilidade deste vírus simplesmente vir a desaparecer um dia (Akpan \& Jaggard, 2020).

A OMS está continuamente monitorando e atualizando as informações sobre a COVID-19, como, por exemplo, como o vírus se propaga nos diferentes continentes, como afeta as pessoas, investimentos realizados para pesquisas com divulgação dos resultados dos estudos clínicos para o controle e tratamento da doença no mundo. A maioria das pessoas infectadas com o vírus experimentará a doença respiratória leve à moderada, sem a necessidade de tratamento especial. Os idosos e aqueles com doenças pré-existentes como: doenças cardiovasculares, hipertensão, diabetes, doenças respiratórias crônicas, doenças auto imunes e câncer, têm maior probabilidade de desenvolver doenças graves. Pensando dessa forma, a melhor maneira de prevenir e desacelerar a transmissão é estar bem informado sobre o mecanismo fisiopatológico, diagnóstico e tratamento da COVID-19. (Moura, 2020).

Diariamente, sites governamentais do Brasil e do mundo divulgam em tempo real dados referentes à situação dos países atingidos pela pandemia da COVID-19 para que toda a comunidade e cientistas possam obter informações de forma transparente e por acesso livre. A situação epidemiológica atualizada por país, território e área está disponível nos sítios eletrônicos https://covid19.who.int/table e https://covid.saude.gov.br. Em 4 de fevereiro de 2021, a partir de 10h12 CET, no Painel do WHO Coronavírus Disease (COVID-19), foi notificado à OMS, 103.631 .793 casos confirmados de COVID-19 no mundo, incluindo 2.251.613 mortes (WHO, 2021a).

Segundo dados extraídos do site do Worldometer, o coronavírus está afetando 219 países e territórios. A lista de países e sua classificação regional deste site é baseada na fonte da Geo Scheme das Nações Unidas. Sendo assim, na última atualização, em 04 de fevereiro de 2021, 25.850 .568 dos pacientes atualmente infectados, 25.888 .928 (99,6\%) estão em condições moderadas e $106.270(0,4 \%)$ descritos como estado de saúde sério ou crítico. Os Casos quetiveram um resultado, do total de 79.284.039, foram considerados como Recuperado /Descarregado ,76.855.104 (97 \%) e 2.284.305 (3\%) Mortes (Worldometer, 2021).

Os casos notificados à OMS, em 4 de fevereiro de 2021 às 13 horas foram distribuídos da seguinte forma: Américas: 46.197.580; Europa: 35.003.091; Sudeste da Ásia: 12.956.439; Mediterrâneo oriental: 5.759.214; África: 2.616.892 e no Pacífico Ocidental:1.455.939 conforme apresentado na Figura 1 a seguir: 
Figura 1 - Dados da distribuição mundial dos casos confirmados de COVID-19 por região (WHO, 2021a).

Painel do WHO Coronavirus Disease (COVID-19)

Última atualizaçăo dos dados: 2021/2/4, 10:12 CET

\section{Situação por região da OMS}

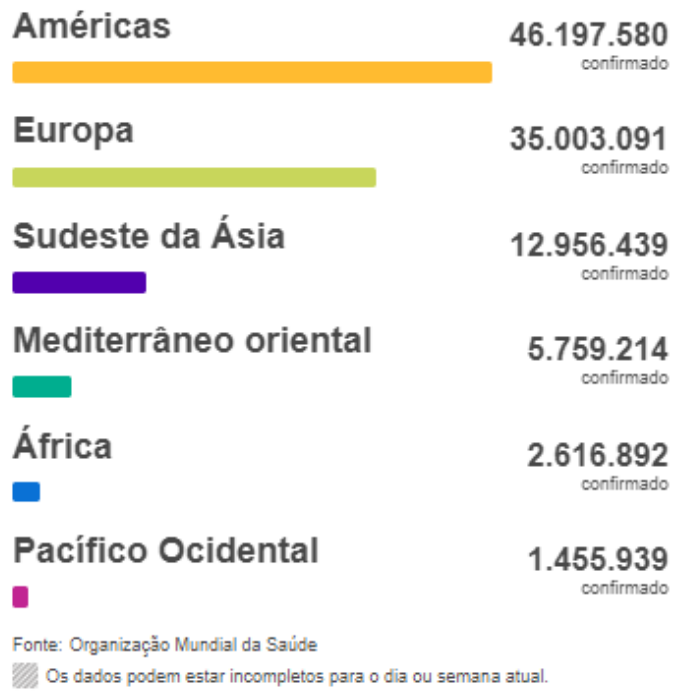

Fonte: Organização Mundial da Saúde: dados extraídos do Painel do WHO: CORONAVÍRUS Disease (COVID-19), atualizados no momento da construção do estudo em 2021/2/4, no site: https://covid19.who.int/.

A partir da análise dos dados foi possível compreender como está a situação dos países mais acometidos pela COVID19. Outro dado importante divulgado atualmente é de que nos Estados Unidos com 27.162.000, está concentrado o maior número de casos no mundo, seguido da Índia com 10.802.458 e, em terceiro lugar, encontra-se o Brasil com 9.354.609 total de casos. Outros países da América do Sul, estão apresentando maior números de casos nos últimos meses, como, por exemplo, Colômbia com 2.125.622, Argentina com 1.952.744, México com 1.886.245, o Peru com 1.158.337 de casos, seguido pela Chile 740.237, Bolívia 222.447, Costa Rica com 195.537, Paraguai com 135.229, Venezuela com 128.315 e Uruguai com 43.215 casos confirmados, o que representa o menor número de pessoas infectadas na América do Sul (Wordometer, 2021).

Com relação à evolução da doença no território brasileiro, o Brasil confirmou o primeiro caso da infecção pelo novo coronavírus, no dia 26 de fevereiro de 2020. Trata-se de um homem de 61 anos, morador da cidade de São Paulo, que esteve na Itália, na região da Lombardia, à trabalho, sozinho, no período de 9 a 21 de fevereiro deste ano. No dia 23 de fevereiro, ele apresentou sinais e sintomas compatíveis com a doença causada pelo novo coronavírus: febre, tosse seca, dor de garganta e coriza. Em 25 de fevereiro, ele procurou atendimento médico no Hospital Israelita Albert Einstein, que registrou então a notificação de caso suspeito. No atendimento, foram adotadas medidas preventivas para transmissão por gotículas, feita coleta de amostras e realizados testes para vírus respiratórios comuns e o exame específico para diagnóstico, conforme preconizado pela OMS (OPAS, 2020a).

O Ministério da Saúde do Brasil, em 20 de março de 2020, com a portaria número 454, declara em todo o território nacional, o estado de transmissão comunitária do coronavírus e destaca algumas medidas para contenção de sua transmissibilidade como: isolamento domiciliar pelo período máximo de quatorze dias para a pessoa com sintomas respiratórios incluindo para seus familiares que residam no mesmo endereço, ainda que estejam assintomáticos, além de oportunizar manejo adequado dos casos leves na rede de atenção primária à saúde e dos casos graves na rede de urgência/emergência. De acordo com a portaria, são considerados sintomas do novo coronavírus: tosse seca, dor de garganta ou dificuldade respiratória, 
acompanhada ou não de febre. Além dos sintomas, o isolamento também depende de prescrição médica, razão pela qual pessoas com sintomas devem procurar um médico para verificar o estado de saúde e confirmar a orientação (Brasil, 2021d).

Ainda de acordo com o site do Worldometer (2021) em 4 de fevereiro até 13 horas no Brasil ocorreram 9.339.921, casos confirmados de COVID-19, com 8.236.864 recuperados e 227.592 óbitos. Dos 889.862 pacientes atualmente infectados, 881.544 (99.1\%) estão em condições moderadas e 8.318 (0.9\%) em estado sério ou crítico. O Ministério da Saúde, através do painel COVID-19 do Conselho Nacional de Secretários de Saúde, (CONASS), apresenta nessa data os seguintes indicadores: Taxa de Letalidade em torno de 2,4\%, Taxa de Mortalidade 108.3100 .000 hab.) e Taxa de incidência de 4.444.2 (100.000 hab.). Quando se observa o painel do CONASS por região, no Sudeste apresenta o número de casos de 3.390.067 com registro de 105.121 óbitos, sendo que São Paulo tem 1.807 .009 casos confirmados, 53.704 óbitos e o Rio de Janeiro, 529.525 casos com 30.172 óbitos (CONASS, 2021). Tal situação pode ser decorrência das características específicas da pandemia: as elevadas taxas de transmissão da doença, em grande parte por pessoas assintomáticas - cada pessoa contamina, antes da adoção de medidas de distanciamento social no Brasil, uma média de três a quatro pessoas, levando ao aumento exponencial de casos ; a gravidade de uma expressiva parcela dos casos, com demanda de hospitalização para cerca de 19\% dos sintomáticos, sendo que 1/4 deles necessitava de internação em Unidade de Terapia Intensiva (UTI) e uso de ventilação mecânica, além de um tempo prolongado de ocupação de leitos hospitalares (Seixas, 2021).

De acordo com Werneck \& Carvalho (2020) quando afirmam que a pandemia da COVID-19 surgiu quando a população brasileira estava vulnerável, com o sistema de saúde à beira do colapso expresso geralmente pela falta de leitos e equipamentos hospitalares, assim como insuficiência de profissionais de saúde capacitados, com ou sem sintomatologia compatível com a COVID-19. As altas taxas de desemprego, cortes de verbas destinadas às políticas sociais, redução de gastos públicos e políticas econômicas interferiram nos investimentos em saúde e na pesquisa. É justamente nesses momentos de crise que a sociedade percebe a importância do investimento em pesquisa, ciência e tecnologia que reverberam na assistência qualificada nos serviços de saúde.

\subsection{Impacto irreversível da pandemia nos Profissionais de saúde: Mortes por COVID-19}

De acordo com Sanchez (2020) com o avanço da doença, agrava-se os índices de letalidade e mortalidade nos profissionais de saúde, mesmo em países que contam com sistemas de saúde relativamente estruturados. Frente a isto, a Aliança Mundial das Profissões da Saúde (WHPA, 2020), que representa 31 milhões de profissionais de saúde no mundo, em carta dirigida aos governos conclamaram que é necessário a priorização de estratégias direcionadas para o apoio às equipes de saúde que estão na linha de frente contra a COVID-19.

Estudo divulgado recentemente apontou que quase 600 profissionais de saúde morreram da COVID-19, incluindo zeladores de hospitais, administradores e trabalhadores de casas de repouso. Relatórios divulgados pelos órgãos de Saúde sobre os desafios que os profissionais de saúde estão enfrentando durante a pandemia, inferem a preocupação quanto aos equipamentos de proteção individual, no que tange a reutilização de máscaras em meio à escassez e equipamentos difundidos. (Jewett, Bailey \& Renwick,2020).

Em Florença, na Itália, na data de 13 de julho de 2020, o governo reconheceu o sacrifício de médicos, enfermeiros e profissionais de saúde que perderam a vida para salvar milhares de pessoas, destacando que morreram 172 médicos de diversas especialidades e 40 enfermeiros. Nesse sentido, inaugurou um local de memória coletiva: vive e luta contra a COVID-19. (FNOMCeO, 2020).

Reportando para o Brasil, o COFEN em maio registrou no Brasil 157 mortes da COVID-19 entre enfermeiros, técnicos e auxiliares de enfermagem. A agência disse que a tendência é que as mortes entre os profissionais de saúde continuem aumentando e alertou que a escala depende de vários fatores, incluindo o fornecimento de equipamentos de proteção e a 
disseminação do vírus entre a população em geral. O Brasil registrou cerca de 411.000 infecções e 25.000 mortes por pandemia, de longe o país mais afetado da América Latina (Kelly, 2020).

Segundo informações do Sindicato dos Médicos de São Paulo (SIMESP) e do Conselho Federal de Enfermagem (COFEN), até o dia 17 de junho de 2020, o Brasil era o país do mundo com maior número de mortes de médicas e médicos (ao todo 139 profissionais) e de enfermeiras e enfermeiros (ao todo 190 profissionais) pela COVID-19. Apesar das dificuldades de acesso aos dados, com prováveis subnotificações, somos possivelmente o país do mundo com maior mortalidade de profissionais da saúde pela COVID-19 (ABRASCO, 2020). O COFEN vem contabilizando, entre casos positivos e suspeitos, mais de 23 mil afastamentos de profissionais de enfermagem desde o início da crise sanitária, sendo o Rio de Janeiro o estado mais afetado pelo coronavírus entre os trabalhadores que atuam no combate à COVID-19, o Estado de São Paulo, em segundo lugar e Mato Grosso do Sul segue com os menores indicadores(Cruz, 2020).

Dando continuidade, o COFEN em maio registrou no Brasil 157 mortes da COVID-19 entre enfermeiros, técnicos e auxiliares de enfermagem. A agência disse que a tendência é que as mortes entre os profissionais de saúde continuem aumentando e alertou que a escala depende de vários fatores, incluindo o fornecimento de equipamentos de proteção e a disseminação do vírus entre a população em geral. O Brasil registrou cerca de 411.000 infecções e 25.000 mortes por pandemia, de longe o país mais afetado da América Latina (Kelly, 2020).

Em janeiro de 2021, podemos observar que o Brasil representou um terço do total de mortes pela COVID-19 entre profissionais de saúde, um dado emergencial tendo em vista que com o grande desfalque do quantitativo de pessoal salvar vidas diariamente se torna uma atividade hercúlea. O dado mais atual sobre a mortalidade da COVID-19 entre trabalhadores da saúde foi divulgado em novembro de 2020 pelo Conselho Internacional da categoria e até o momento o número de mortos em 44 países era de 1.500, a cifra já deve ter sido ultrapassada. Jorge Howard Catton afirma que o fato de o número de enfermeiros e enfermeiras mortos pela COVID-19 ser semelhante ao número de mortos na I Guerra Mundial é alarmante (CNTS, 2021).

Segundo o COFEN (2021), desde o início da pandemia, a doença foi responsável pelo aumento da taxa de mortalidade dos profissionais de enfermagem, afetando todas as categorias (enfermeiras, técnicos, auxiliares de enfermagem e obstetrizes) que atuavam na linha de frente na assistência aos pacientes. Nos dados divulgados pelo Ministério da Saúde do número de casos de óbitos no país, dos 200.000 mil decorrentes da Covid 19, cerca de 500 óbitos eram dos profissionais de Enfermagem. De acordo com os dados do Conselho Federal de Enfermagem, cerca de 30 profissionais morreram na primeira semana de 2021 (COFEN, 2021a).

O portal observatório da Enfermagem, criado pelo Conselho Federal de Enfermagem, contabiliza diariamente o número de profissionais contaminados pelo coronavírus. Dessa forma, até o dia 09 de fevereiro de 2021 temos o número de 47.869 profissionais infectados, 553 óbitos e uma taxa de letalidade de 2,01\%. Diante desses números é notória a importância da constante fiscalização e vigilância por parte das entidades de Enfermagem, assim como os Gestores do Sistema Único de Saúde e da iniciativa privada, assegurando melhores condições de trabalho para esses profissionais de enfermagem exercerem suas atividades de forma segura (COFEN, 2021b).

Ressalta-se que apesar dos dados estatísticos publicados sobre os problemas diários de contaminação, agravos e óbitos ocorridos. O COFEN precisou recorrer à Justiça em busca do direito de afastamento e à testagem dos profissionais, reforçando a importância da representatividade e as dificuldades inerentes à essa classe trabalhadora que é reconhecidamente o maior quantitativo nos serviços de saúde.

O Brasil responde por 40\% das mortes globais de enfermeiros pela COVID-19, e existem mais de 15.000 enfermeiros infectados com coronavírus no país da América do Sul, que já causaram mais de 18 mil mortes. De acordo com Walquirio Almeida, porta-voz do COFEN, os enfermeiros infectados representam quase $40 \%$ de casos no mundo, e essas mortes são vinculadas com a escassez de recursos humanos e materiais adequados. Dando continuidade Walquirio afirma que: "Os números 
são muito preocupantes, não esperávamos tantos", e "A situação só melhora se as autoridades tomarem ações efetivas e rápidas". A nação de 210 milhões de pessoas se tornou um epicentro da pandemia atrás apenas da Rússia e dos Estados Unidos em número de casos. As infecções vêm quebrando recordes quase diariamente (Kelly, 2020).

A morte dos profissionais de saúde sinaliza a negligência do poder público com a situação de trabalho e assistência à saúde. De acordo com Manoel Neri, o Coren recebeu mais de 5 mil denúncias, grande parcela referente a escassez e equipamentos de proteção individuais (EPIs) inadequados. Mostra-se fundamental o afastamento dos profissionais do grupo de risco da linha de frente, de fato precisamos entender que esses profissionais são seres humanos e não máquinas (Coren, 2020).

Para Shanafelt, Ripp \& Trockel (2020) manter uma força de trabalho de saúde adequada nesta crise requer não apenas um número adequado de profissionais de saúde como também qualidade técnica científica e capacidade de trabalhar em situação pandêmica o que leva alto desgaste físico e emocional. Visto que os surtos em pacientes criticamente enfermos podem durar de semanas a meses, também é essencial que os profissionais de saúde sejam capazes de desempenhar todo o seu potencial durante um intervalo de tempo prolongado. Ao mesmo tempo que lidam com as mudanças sociais e estressores emocionais enfrentados por todas as pessoas, os profissionais de saúde enfrentam maior risco de exposição, cargas de trabalho extremas, dilemas morais e um ambiente de prática em rápida evolução que difere muito do que eles estão familiarizados.

\subsection{A ciência à serviço da humanidade: a busca pela vacina.}

Com o avanço da doença, aumento do número de óbitos e agravos decorrentes da pandemia da COVID-19 no mundo, a OMS e seus parceiros aceleraram o desenvolvimento de vacinas da COVID-19, sem perder de vista os padrões de segurança. No passado, as vacinas eram desenvolvidas por meio de uma série de etapas, incluindo vários testes clínicos sobre sua eficácia e eficiência, o que demanda vários anos para sua ampla utilização. Atualmente, não há evidência de que as vacinas existentes aprovadas para outras doenças possam também proteger contra COVID-19. No entanto, já existem estudos que algumas vacinas existentes - como a vacina Bacille Calmette-Guérin (BCG), que é usada para prevenir a tuberculose - também são eficazes para COVID-19. A WHO (2021b) estará avaliando todas as evidências desses estudos, quando disponíveis.

Diante da gravidade da pandemia e ausência de medicamentos para cura, a única solução urgente para a humanidade são as vacinas contra a COVID-19. Assim, investimentos financeiros e colaborações científicas, sem precedentes, estão mudando a forma como as vacinas são desenvolvidas. Isso significa que algumas etapas do processo de pesquisa e desenvolvimento vêm ocorrendo em paralelo, o que não torna os estudos menos rigorosos, pois eles seguem rígidos padrões clínicos e de segurança. Por exemplo, alguns ensaios clínicos, em diferentes países, estão avaliando vários tipos de vacinas ao mesmo tempo, o que pode ser difícil para a comunidade médica e os voluntários em potencial estimar adequadamente os riscos potenciais de participar de um estudo de desafio humano (WHO, 2021c).

No mundo científico, há um consenso que a crise só vai acabar de fato quando houver vacina para todos. Cientistas estão desenvolvendo muitas vacinas potenciais para COVID-19. Todas essas vacinas são projetadas para ensinar o sistema imunológico a reconhecer e bloquear com segurança o vírus. O detalhamento da produção e estudos em desenvolvimento encontra-se descrito no Relatório Técnico de Monitoramento de Vacinas em Desenvolvimento contra SARS-CoV-2, da Secretaria de Ciência, Tecnologia, Inovação e Insumos Estratégicos em Saúde (Brasil, 2021f). Das vacinas candidatas em estudos clínicos, 20 encontravam-se na fase III de ensaios clínicos para avaliação de eficácia e segurança, a última etapa antes da aprovação pelas agências reguladoras e posterior imunização da população (WHO, 2021c)

Até 12 de janeiro de 2021 a OMS (2021) relatou 173 vacinas contra a COVID-19 candidatas em fase pré-clínica de pesquisa e 63 vacinas candidatas em fase de pesquisa clínica. Atualizações sobre as fases de vacinas em desenvolvimento encontram-se disponíveis no sítio eletrônico (WHO, 2021c). Vários tipos diferentes de vacinas potenciais para COVID-19 estão 
em estudo clínico de fase III, sendo destacadas, a seguir, as principais em desenvolvimento no Plano Nacional de Operacionalização da Vacinação Contra a COVID-19 (Brasil, 2021c):

a) Vacinas de vírus inativados - As vacinas de vírus inativados utilizam tecnologia clássica de produção, através da qual é produzida uma grande quantidade de vírus em culturas de células, sendo estes posteriormente inativados por procedimentos físicos ou químicos. Geralmente são vacinas seguras e imunogênicas, pois os vírus inativados não possuem a capacidade de replicação e assim o organismo não fica exposto às grandes quantidades de antígenos. As vacinas COVID-19 de vírus inativados em fase III são desenvolvidas por empresas associadas aos institutos de pesquisa Sinovac, Sinopharm/Wuhan Institute of Biological Products, Sinopharm/ Beijing Institute of Biological Products, Bharat Biotech, Research Institute for Biological Safety Problems e Chinese Academy of Medical Sciences.

b) Vacinas de vetores virais - Estas vacinas utilizam vírus humanos ou de outros animais, replicantes ou não, como vetores de genes que codificam a produção da proteína antigênica (no caso a proteína Spike ou proteína S do SARS-CoV-2). Os vetores virais replicantes podem se replicar dentro das células enquanto os não replicantes, não conseguem realizar o processo de replicação, porque seus genes principais foram desativados ou excluídos. Uma vez inoculadas, estas vacinas com os vírus geneticamente modificados estimulam as células humanas a produzir a proteína Spike, que vão, por sua vez, estimular a resposta imune específica. $\mathrm{O}$ vírus recombinante funciona como um transportador do material genético do vírus alvo, ou seja, é um vetor inócuo, incapaz de causar doenças. As vacinas em fase III que utilizam essa plataforma são: Oxford/AstraZeneca (adenovírus de chimpanzé); CanSino (adenovírus humano 5 - Ad5); Janssen/J\&J (adenovírus humano 26 - Ad26) e Gamaleya (adenovírus humano 26 - Ad26 na primeira dose, seguindo de adenovírus humano 5 - Ad5 na segunda dose). 18 Vacinas baseadas em proteínas, que usam fragmentos inofensivos de proteínas ou cascas de proteínas que imitam o vírus COVID-19 para gerar com segurança uma resposta imune.

c) Vacina de RNA mensageiro - O segmento do RNA mensageiro do vírus, capaz de codificar a produção da proteína antigênica (proteína Spike), é encapsulado em nanopartículas lipídicas. Da mesma forma que as vacinas de vetores virais, uma vez inoculadas, estas vacinas estimulam as células humanas a produzir a proteína Spike, que vão por sua vez estimular a resposta imune específica. Esta tecnologia permite a produção de volumes importantes de vacinas, mas utiliza uma tecnologia totalmente nova e nunca antes utilizada ou licenciada em vacinas para uso em larga escala. Atualmente, as vacinas produzidas pela Moderna/NIH, Pfizer/BioNTec e CureVac AG são as duas vacinas de mRNA em fase III. Do ponto de vista de transporte e armazenamento, estas vacinas requerem temperaturas muito baixas para conservação $\left(-70^{\circ} \mathrm{C}\right.$ no caso da vacina candidata da Pfizer e $-20^{\circ} \mathrm{C}$ no caso da vacina candidata da Moderna), o que pode ser um obstáculo operacional para a vacinação em massa, especialmente em países de renda baixa e média.

d) Unidades proteicas - Através de recombinação genética do vírus SARS-CoV-2, se utilizam nanopartículas da proteína Spike (S) do vírus recombinante SARS-CoV-2 rS ou uma parte dessa proteína denominada de domínio de ligação ao receptor (RDB). Os fragmentos do vírus desencadeiam uma resposta imune sem expor o corpo ao vírus inteiro. Esta é uma tecnologia já licenciada e utilizada em outras vacinas em uso em larga escala e, usualmente, requer adjuvantes para indução da resposta imune. As vacinas COVID-19 que utilizam esta tecnologia em fase III são a vacina da Novavax, que utiliza como adjuvante a Matriz-M1TM, e a vacina desenvolvida pela "Anhui Zhifei Longcom Biopharmaceutical”, "Institute of Microbiology, Chinese Academy of Sciences" e "Clover Biopharmaceuticals.

O impacto das vacinas da COVID-19 na pandemia dependerá de vários fatores como a sua eficácia; com que rapidez elas serão aprovadas, fabricadas, distribuídas e utilizadas em toda a população; e quantas pessoas serão vacinadas em todo o 
mundo. A maioria dos cientistas prevê que, como a maioria das outras vacinas, as vacinas COVID-19 não serão 100\% eficazes e seguras. $\mathrm{O}$ fato é que o mundo científico nunca esteve tão unido para trabalhar e ajudar a garantir que todas as vacinas aprovadas sejam tão eficazes quanto possível, para que possam ter o maior impacto sobre a pandemia (WHO, 2021c).

Greshko (2021) destaca que segundo os especialistas a trajetória pós-pandêmica do SARS-CoV-2 vai depender de três fatores essenciais: período em que as pessoas vacinadas ou contaminadas retêm a imunidade ao vírus, a rapidez com que o vírus evolui e o quão amplamente as populações mais velhas se tornam imunes durante a própria pandemia. Dependendo dos resultados destes três fatores, o mundo pode vir a enfrentar vários anos de uma lenta transição pós-pandêmica - uma transição marcada por uma evolução viral contínua, surtos localizados e possivelmente várias dosagens de vacinação atualizadas serão necessárias.

De acordo com a WHO (2021c) as instituições estão trabalhando em colaboração com cientistas, empresas e organizações globais de saúde por meio do ACT Accelerator. Quando uma vacina segura e eficaz for encontrada, a COVAX (liderada pela OMS, GAVI e CEPI) facilitará o acesso e distribuição equitativos dessas vacinas para proteger as pessoas em todos os países. Recomenda-se que todos os países possam ter um plano de gestão para tão logo sejam aprovadas vacinas seguras e eficazes de forma justa, as pessoas em maior risco, como os profissionais de saúde e idosos em situação vulnerável sejam priorizados, sendo essencial dar continuidade às ações de saúde pública para suprimir a transmissão e reduzir os danos e as taxas de mortalidade causadas pela doença.

Sendo assim, no início do ano de 2021, cerca de 50 países já aplicaram mais de 38 milhões de doses de vacinas. Sendo o imunizante da Pfizer/BioNTech o mais utilizado. Em números absolutos os Estados Unidos é o estado que mais vacinou até o momento, seguido da China e Reino Unido. Em números relativos Israel e Emirados Árabes e Bahrein se destacam. No mundo, o planejamento da imunização começou com grupos que têm mais contato com SARS-CoV-2 ou são mais vulneráveis aos efeitos da doença, como profissionais de saúde e idosos atendendo às recomendações da OMS (Gaglioni, 2021).

O fato é que o SARS-CoV-2 propagou-se rapidamente no mundo inteiro e, com cada nova replicação, há a probabilidade de mutações com maior poder de virulência e transmissibilidade. No futuro, a médio e longo prazo, o impacto do coronavírus dependerá das variadas mutações e evolução do vírus, mas, por enquanto, a vacinação e a vigilância são essenciais para terminar sua fase pandêmica. O sistema imunitário humano, ao mesmo tempo que protege muitos de nós de doenças graves, também atua como um cerne evolutivo, pressionando o vírus na seleção de mutações que o tornam mais eficazes na ligação com as células humanas. Nos próximos anos irão revelar o quão bem o nosso sistema imunitário consegue acompanhar essas alterações. As novas variantes do SARS-CoV-2 reforçam que a vacinação deve ser generalizada e que as outras medidas de combate à transmissão, como o uso de máscara e o distanciamento social, continuam sendo essenciais, pois quanto menos o vírus se propagar, menos oportunidades tem para evoluir (Greshko, 2021).

\subsection{Imunização dos profissionais de saúde}

Por trás do interesse das farmacêuticas internacionais com o Brasil, está o fato de o país oferecer um item essencial na elaboração de toda vacina: pessoas em situação de alto risco de contágio pela doença. Só atrás dos Estados Unidos em casos confirmados e mortes pela COVID-19. O Brasil tornou-se um campo fértil para testes de tratamentos contra a doença. É um prêmio de consolação por não ter conseguido controlar o avanço da pandemia desde o início (Branco, 2020).

Na tarde do dia 17 de janeiro de 2021 a Anvisa aprovou, por unanimidade, o uso emergencial das vacinas Coronavac e Oxford contra a COVID-19, apesar do Brasil enfrentar momentos de tensões políticas e um negacionismo da ciência. A primeira pessoa a receber a dose da Coronavac foi a enfermeira do Instituto de Infectologia Emílio Ribas Mônica Calazans, de 54 anos (Meneses, 2021). A partir do dia 18 de janeiro de 2021 foi iniciada a vacinação no Brasil, em vista de viabilizar as ações de vacinação o plano nacional de operacionalização, optou-se pela seguinte ordem: preservação do funcionamento dos serviços de saúde, proteção dos indivíduos com maior risco de desenvolvimento de formas graves e óbitos, seguido da 
preservação do funcionamento dos serviços essenciais e proteção dos indivíduos com maior risco de infecção (Brasil, 2021a). Sendo os profissionais de saúde a classe trabalhadora incluída no grupo prioritário. Apesar disso, devido à quantidade limitada de doses, o Governo propôs a seguinte priorização para os profissionais de saúde, que poderá ser adaptada por cada estado e município, representada no Quadro 1 (Brasil, 2021b):

Quadro 1: Recomendação da ordem de priorização dos profissionais de saúde.

\section{ORDEM DE PRIORIZAÇÃO DA IMUNIZAÇÃO DOS PROFISSIONAIS DE SAÚDE}

Profissionais envolvidos nas equipes de vacinação dos grupos elencados para as 6 milhões de doses.

Trabalhadores das instituições de longa permanência de idosos e de residências inclusivas (Serviço de Acolhimento Institucional em Residência Inclusiva para jovens e adultos com deficiência);

Profissionais que trabalham nos serviços de saúde públicos e privados, tanto da urgência quanto da atenção básica, envolvidos diretamente na atenção/referência para os casos suspeitos e confirmados de COVID-19;

Por fim, se ainda houver doses disponíveis, demais trabalhadores de saúde.

Fonte: Elaboração das autoras baseado no Informe do Ministério da saúde (Brasil,2021b).

Para atender essa nova necessidade e suprir os Estados brasileiros com recursos necessários para vacinação, o Ministério da Saúde junto com o Conselho Nacional de Secretarias Municipais de Saúde lançaram um programa de educação continuada com o intuito de capacitar 94 mil profissionais de saúde que atuam na linha de frente dos serviços de imunizações, intitulado como ImunizaSUS. A proposta é adaptar salas das Unidades Básicas de Saúde em salas e aula de ensino a distância (EaD), com carga horária de 180 horas, visando a segurança dos profissionais de saúde e também da população que receberá a vacina (Nascimento, 2021).

\section{Conclusão}

O Brasil e o mundo experimentaram o efeito devastador do um novo vírus, causador da COVID-19. Uma doença que ao longo de sua evolução apresentou vários desdobramentos, que exigiu das organizações de saúde implementar estratégias e políticas públicas, em curto prazo, no gerenciamento e articulação de ações e estratégias para deter a disseminação do vírus. Outro aspecto da pandemia da COVID-19 é que ela envolve a adesão de medidas de segurança e de proteção, com mudanças de comportamento, individual e coletiva, o que influencia diretamente no seu impacto sobre a população.

Corrobora-se com Oliveira et al (2020) quando afirmam que a pandemia da COVID-19 expõe as fragilidades estruturais e os pontos de estrangulamento dos sistemas de saúde nos diferentes países, em particular, a distribuição desigual de profissionais da saúde e de infraestrutura dos serviços disponíveis na atenção de média e alta complexidade. Assim como, a capacidade limitada de produção, realização insuficiente de testes de diagnósticos e dificuldade para o acesso às vacinas disponíveis. No Brasil, os desafios para o combate da pandemia são imensos e agravados pelo contexto social, que impõe condições de vida e de saúde precárias, especialmente, à população mais carente e vulnerável, residente nas periferias dos grandes centros urbanos .

No combate da pandemia, ficou evidente a necessidade de maior investimento nas tecnologias de saúde e em pesquisas para detectar novas patologias que possam acometer a humanidade, reforçando a importância do fortalecimento das parcerias das grandes instituições de saúde para criação de medidas e protocolos voltados unicamente para a preservação e proteção da vida humana. Conforme estudos divulgados, nos meios de comunicação nacional e internacional, um dos impactos positivos da 
pandemia para a população, foi tornar visível a importância da solidariedade e união de esforços para a valorização do cuidado humanitário oferecido pelos profissionais de saúde. Nunca se falou tanto da importância e das dificuldades enfrentadas pelos enfermeiros e demais profissionais no processo de cuidar. O mundo pode conhecer e debater os aspectos da assistência e compreender que até nos países considerados "ricos", há deficiência de profissionais de saúde e os recursos destinados para o gerenciamento das adversidades nos sistemas de saúde são limitados.

Nota-se as consequências da COVID-19 para a saúde dos profissionais, pois ficou evidente o sofrimento físico e mental e suas sequelas. No decorrer e avançar dos casos confirmados entre os profissionais de saúde, as causas identificadas para o acometimento e óbito foram variadas como: sobrecarga e exaustão do trabalhador, falta de profissionais para atender as demandas, ausência ou redução dos equipamentos de proteção individual, baixa qualificação, testagem insuficiente dos profissionais, falta de capacitação para atuar nos estágios avançado da doença, dentre outras. A classe da saúde nunca mais será a mesma e a pandemia e suas repercussões ainda se estenderão por um longo tempo.

Agora os profissionais de saúde estão em um novo momento e a partir desse momento novas estratégias de enfrentamento foram aprimoradas e desenvolvidas. Diante do exposto, os gestores dos serviços de saúde, devem priorizar a segurança dos profissionais envolvidos diretamente no cuidado, seja do administrativo ao assistencial, com a intenção de prevenir a recorrência dos agravos e injúrias decorrentes dessa nova pandemia. Acredita-se que o impacto social, científico, econômico e humanitário terá proporções globais ainda incalculáveis nas próximas décadas, apesar dos investimentos nas tecnologias e uso emergencial das vacinas para imunização da população.

Para futuros estudos, espera-se o desenvolvimento de pesquisas que ofereçam subsídios para o fortalecimento do conhecimento científico, referente ao impacto deixado pela COVID-19 na segurança da população e dos profissionais de saúde e a resposta da imunização da população a curto, médio e longo prazo. Espera-se que a amplificação e realização de novas pesquisas forneçam resultados e respostas capazes de fortalecer a proteção da população acometida pela doença nos diversos contextos e cenários de saúde.

\section{Referências}

Associação Brasileira de Saúde Coletiva. (2020). Em defesa da vida: solidariedade aos profissionais de saúde mortos por COVID-19. ABRASCO. https://www.abrasco.org.br/site/noticias/posicionamentos-oficiais-abrasco/em-defesa-da-vida-solidariedade-aos-profissionais-de-saude-mortos-por-COVID$19 / 49520 /$.

Akpan, N., \& Jagguard, V. (2020). Anthony Fauci: Não existem Provas Científicas de que o Coronavírus Foi Criado num Laboratório Chinês. NatGeo. https://www.natgeo.pt/ciencia/2020/05/anthony-fauci-nao-existem-provas-cientificas-de-que-o-coronavirus-foi-criado-num.

Alessi, G. (2020). Brasil já perdeu mais profissionais de enfermagem para o coronavírus do que Itália e Espanha juntas. EL PAÍS. https://brasil.elpais.com/brasil/2020-05-06/brasil-ja-perdeu-mais-profissionais-de-enfermagem-para-o-coronavirus-do-que-italia-e-espanha-juntas.html.

Alessi, G. (2021). Brasil responde por um terço das mortes globais entre profissionais de enfermagem por COVID-19. EL PAÍS. https://brasil.elpais.com/brasil/2021-01-08/brasil-responde-por-um-terco-das-mortes-globais-entre-profissionais-de-enfermagem-por-COVID-19.html.

Astor, M., Barnes, B., Barrow, K., Blinder, A., Cochrane, E., Cook, L. R., Cooper, M., Cowan, J., Das, A., Fandos, N., Frey tas-Tamura, K., Furber, M., Gold, M., Green, E. L., Gross, J., Hamby, C., Haberman, M., Hadi,M., Harmon, A., Hartocollis, A., Hu, W., Jacobs,J., Levenson, M., Mervosh, S., Miller, C. C., Phillips, M., Rabin, R. C., Schmidt, M. S., Smith, M., Soto, K., Steinhauer, J., Stevens, M., Sullivan, E., Vigdor, N., Waldstein, D., Witz, B., \& Zimmer, C. (2020). Quatro meses após o primeiro caso, número de mortos nos EUA ultrapassa 100.000. The New York times. https://www.nytimes.com/2020/05/27/us/coronavirus-live-news-updates.html\#link-30ec6ebb.

Branco, L. (2020). Brasil Fecha Acordo Com Universidade De Oxford Para Testar Vacina Contra COVID-19. Revista Época - Globo. https://epoca.globo.com/brasil-fecha-acordo-com-universidade-de-oxford-para-testar-vacina-contra-COVID-19-24503480.

Brasil (2020). Ministério da Saúde. COVID-19 NO BRASIL. https://susanalitico.saude.gov.br/extensions/COVID-19_html/COVID-19_html.html.

Brasil (2021a). Ministério da Saúde. Informe Técnico: Campanha Nacional de Vacinação contra a COVID-19.

Brasil (2021b). Ministério da Saúde. Plano nacional de operacionalização da vacinação contra a COVID-19.

Brasil (2021c). Ministério da Saúde. Secretaria de Vigilância em Saúde Departamento de Imunização e Doenças Tran smissíveis Coordenação-Geral do Programa Nacional de Imunizações. Plano nacional de operacionalização da vacinação contra a covid-19. 2ª edição | Brasília/DF 22/01/2021. 90 p. 
Brasil (2021d). Ministério da Saúde. Portaria no 454 Dispõe sobre a declaração, em todo o território nacional, o estado de transmissão comunitária do coronavírus (COVID-19). Diário Eletrônico, Órgão: Ministério da Saúde/Gabinete do Ministro Edição: 55-F|Seção: 1 - Extra|Página: 1. https://www.in.gov.br/en/web/dou/-/portaria-n-454-de-20-de-marco-de-2020-249091587.

Brasil. Ministério da Saúde. (2020e). Entenda a diferença entre Coronavírus, COVID-19 e Novo Coronavírus. https://www.gov.br/pt-br/noticias/saude-evigilancia-sanitaria/2020/03/entenda-a-diferenca-entre-coronavirus-covid- -19-E-novo-coronavirus.

Brasil. Ministério da Saúde. (2021f). Relatórios de Monitoramento (SCTIE). https://www.gov.br/saude/pt-br/Coronavirus/vacinas/relatorios-de-monitoramentosctie.

Conselho Nacional de Secretários de Saúde. (2021). PAINEL CONASS COVID- 19. CONASS. https://www.conass.org.br/painelconasscovid19/.

Chen, K. Y., Yang, C. M., Lien, C. H., Chiou, H. Y., Lin, M. R., Chang, H. R., \& Chiu, W. T. (2013). Burnout, job satisfaction, and medical malpractice among physicians. International journal of medical sciences, 10(11), 1471-1478. https://doi.org/10.7150/ijms.6743.

Conselho Federal de Enfermagem. (2021a). Brasil representa um terço das mortes de profissionais de Enfermagem por covid-19. COFEN. http://www.cofen.gov.br/brasil-responde-por-um-terco-das-mortes-de-profissionais-de-enfermagem-por-covid-19_84357.html.

Conselho Federal de Enfermagem. (2021b). Observatório de Enfermagem. Profissionais infectados com COVID-19 informado pelos enfermeiros responsáveis técnicos/coordenadores. COFEN. http://observatoriodaenfermagem.cofen.gov.br/.

Conselho Regional de Enfermagem. (2020). Brasil responde por 30\% das mortes de profissionais de Enfermagem por COVID-19. COFEN. http://ba.corens.portalcofen.gov.br/brasil-responde-por-30-das-mortes-de-profissionais-de-enfermagem-por-COVID-19_56592.html.

Confederação Nacional dos Trabalhadores na Saúde. (2021). Notícias: coronavírus brasil responde por um terço das mortes globais entre profissionais da enfermagem por covid-19. CNTS. https://cnts.org.br/noticias/brasil-responde-por-um-terco-das-mortes-globais-entre-profissionais-da-enfermagem-por-covid19.

Cruz, P. R. (2020). COVID-19 Já Matou 208 Profissionais De Enfermagem No Brasil, DIZ COFEN. R7 Brasil. https://noticias.r7.com/saude/COVID-19-jamatou-208-profissionais-de-enfermagem-no-brasil-diz-cofen-07072020.

Fagundes, M. (2020). Com 40\% dos casos, Brasil lidera mortes de enfermeiros por COVID-19. Exame. Brasil. https://exame.com/brasil/com-40-dos-casosbrasil-lidera-mortes-de-enfermeiros-por-COVID-19/.

Fagundes, M. (2020). COVID-19 está matando enfermeiras no Brasil mais do que em qualquer outro lugar. BoombergQuint. https://www.bloombergquint.com/amp/onweb/COVID-19-is-killing-nurses-in-brazil-more-than-anywhere-else.

Federazione Nazionale degli Ordini dei Medici Chirurghi e degli Odontoiatri. (2020). A Firenze un luogo della memoria per medici e infermieri morti per Covid. FNOMCeO. https://portale.fnomceo.it/a-firenze-un-luogo-della-memoria-per-medici-e-infermieri-morti-per-covid/.

Fundação Oswaldo Cruz. (2020). Por que a doença causada pelo novo vírus recebeu o nome de COVID-19? Portal FIOCRUZ. https://portal.fiocruz.br/pergunta/por-que-doenca-causada-pelo-novo-virus-recebeu-o-nome-de-COVID-19.

Gaglioni, C. (2021). O estágio da vacinação contra a COVID-19 pelo mundo. NEXO Jornal. https://www.nexojornal.com.br/expresso/2021/01/16/Oest\%C3\%A1gio-da-vacina\%C3\%A7\%C3\%A3o-contra-a-covid-19-pelo-mundo.

Greshko, Michael (2021). A COVID-19 provavelmente ficará conosco para sempre. eis como iremos conviver com a doença. NatGeo. https://www.natgeo.pt/ciencia/2021/01/a-COVID-19-provavelmente-ficara-connosco-para-sempre.

Jewett, C., Bailey, M., \& Renwich, D. (2020). Nearly 600 US health care workers have died of COVID-19. ABC NEWS. https://abcnews.go.com/amp/Health/600-us-health-care-workers-died-COVID-19/story?id=71132667\%204/7.

Jewett, C., \& Szabo, L. (2020). O coronavírus está matando muito mais profissionais de saúde dos eua do que dados oficiais sugerem. The guardian. https://amp.theguardian.com/us-news/2020/apr/15/coronavirus-us-health-care-worker-death-toll-higher-official-data-suggests.

Johns Hopkins University \&Medicine. Coronavirus Resource center (2021). COVID-19 Dashboard by the Center for Systems Science and Engineering (CSSE) at Johns Hopkins University (JHU). Johns Hopkins University \& Medicine. https://coronavirus.jhu.edu/map.html.

Kahn, J., \& Technologies, J.H.P.E.G.D.C.T. (2020). Digital Contact Tracing for Pandemic Response: Ethics and Governance Guidance. Baltimore: Johns Hopkins University Press., doi:10.1353/book.75831.

Kelly, B. (2020). Brasil, el país con más muertes de enfermeros por COVID-19. Diario las Américas. https://www.diariolasamericas.com/america-latina/brasilel-pais-mas-muertes-enfermeros-COVID-19-n4200107/amp.

Lai J, Ma S, Wang Y, Cai Z, Hu J, Wei N, Wu J, Du H, Chen T, Li R, Tan H, Kang L, Yao L, Huang M, Wang H, Wang G, Liu Z, \& Hu S. Factors Associated with Mental Health Outcomes Among Health Care Workers Exposed to Coronavirus Disease 2019. JAMA Netw Open. 2020 Mar 2;3(3):e203976. doi: 10.1001/jamanetworkopen.2020.3976.

Lima, C. M. A. de O. (2020). Informações sobre o novo coronavírus (COVID-19). Radiologia Brasileira, 53(2), V-VI. Epub April 17, 2020.https://doi.org/10.1590/0100-3984.2020.53.2e1.

Costa de Moura, M. L. (2020). Coronavírus e COVID-19. Saúde Coletiva (Barueri), 10(53), 2370-2375. https://doi.org/10.36489/saudecoletiva.2020v10i53p2370-2375

Nascimento, L. (2021). ImunizaSUS capacitará profissionais de saúde para vacinação. Agência Brasil. https://agenciabrasil.ebc.com.br/saude/noticia/202101/imunizasus-capacitara-profissionais. 
Research, Society and Development, v. 10, n. 3, e3410312903, 2021

(CC BY 4.0) | ISSN 2525-3409 | DOI: http://dx.doi.org/10.33448/rsd-v10i3.12903

Oliveira, A. C de., Lucas, T. C., \& Iquiapaza, R. A. (2020). O QUE A PANDEMIA DA COVID-19 TEM NOS ENSINADO SOBRE ADOÇÃO DE MEDIDAS DE PRECAUÇÃO? Texto \& Contexto - Enfermagem, 29, e20200106. Epub May 08, 2020. https://doi.org/10.1590/1980-265x-tce-2020-0106.

Organização Pan-Americana de Saúde (2020a). Brasil, confirma o primeiro caso de infecção pelo novo coronavírus. https://www.paho.org/bra/index.php?option=com_content\&view=article\&id=61 13:brasil-confirma-primeiro-caso-de-infeccao-pelo-novocoronavirus\&Itemid $=812$.

Organização Panamericana de Saúde. Folha informativa COVID-19. (2021a). Escritório da OPAS e da OMS no Brasil. https://www.paho.org/pt/covid19

Organização Pan-Americana de Saúde. (2021b). Doença por coronavírus (COVID-19): Vacinas. https://www.who.int/news-room/q-a-detail/coronavirusdisease-(COVID-19)-vaccines?adgroupsurvey= $\{$ adgroupsurvey $\}$ \&clid=Cj0KCQiAjKqABhDLARIsABbJrGnfWCPxxBGOIW-

sOOJJcpFPI9wG9635nZr_XDItIvWuqP5vJfksdhEaAjEeEALw_wcB.

Pereira, A. S., Shitsuka, D. M., Parreira, F. J., \& Shitsuka, R. (2018). Metodologia da pesquisa científica UFSM. https://repositorio.ufsm.br/bitstr eam/handle/1/15824/Lic_Computacao_Metodologia-Pesquisa-Cientifica.pdf?sequence=1.

Pimentel, R. M. M., Daboin, B. E. G., Oliveira, A. G de., \& Macedo Jr, H. (2020). A disseminação da covid-19: um papel expectante e preventivo na saúde global. Journal of Human Growth and Development, 30(1), 135-140. https://dx.doi.org/10.7322/jhgd.v30.9976.

Sanchez, M. C. O., de Moraes, Érica B., Valente, G. S. C., Braga, A. L. de S., Nassar, P. R. B., \& Xavier, M. L. (2020). Coronavirus pandemic and Primary Care: reflections on the challenges of managers. Research, Society and Development, 9(7), e310974154. https://doi.org/10.33448/rsd-v9i7.4154.

Shanafelt, T., Ripp, J., Trockel, M. Understanding and Addressing Sources of Anxiety Among Health Care Professionals During the COVID-19 Pandemic. JAMA. 2020;323(21):2133-2134. doi:10.1001/jama.2020.5893.

Seixas, C. Terenzi., Merhy, E. E., Feuerwerker, L C. Macruz., Santo, T. B. do E., Slomp Junior, H., \& Cruz, K. T da. (2021). A crise como potência: os cuidados de proximidade e a epidemia pela COVID-19. Interface - Comunicação, Saúde, Educação,25(Suppl. 1), e200379. Epub November 20, 2020.https://doi.org/10.1590/interface.200379.

Severino, A. J. (2017). Metodologia do trabalho científico. (24a ed.), Cortez.

Sociedade Brasileira para o Progresso da Ciência. (2020). Cientistas trabalham até de graça na pesquisa sobre COVID-19. SBPC. http://portal.sbpcnet.org.br/noticias/cientistas-trabalham-ate-de-graca-na-pesquisa-sobre-covid/.

Kawa, H. ., Correia, D. M. da S. ., Kazniakowski, A. W. ., Andrade , B. N. D. de ., Arêas , C. M. ., Silva , J. M. L. da ., Freitas , J. V. de ., Dall'Orto, L. T. C. ., Marinho, L. dos S. S. ., Silva, M. S. de A. e ., Gardengui , M. C. L. ., Lessa, M. P. ., Costa, S. M. da ., Costa , S. P. O., \& Rosa, M. L. G. . (2021). Performance of cities in the metropolitan region of Rio de Janeiro in hospitalizations for Covid-19. SIVEP-Gripe based study. Research, Society and Development, 10(1), e25710111611. https://doi.org/10.33448/rsd-v10i1.11611.

Werneck, G. L., \& Carvalho, M. S. (2020). Editorial. A pandemia de COVID-19 no Brasil: crônica de uma crise sanitária anunciada. Cadernos de Saúde Pública, 36(5), e00068820. Epub May 08, 2020.https://doi.org/10.1590/0102-311x00068820

Worldometers (2021). Pandemia de coronavírus COVID-19. Worldmeters. https://www.worldometers.info/coronavirus/.

World Health Organization. (2020a). Pneumonia of unknown cause - China. http://www.who.int/csr/don/05-january-2020-pneumonia-of-unkown-causechina/en/.

World Health Organization. (2020b). OMS declara emergência de saúde pública de importância internacional por surto de novo coronavírus. https://www.paho.org/bra/index.php?option=com_content\&view=article\&id=6100:oms-declara-emergencia-de-saude-publica-de-importancia-internacionalem-relacao-a-novo-coronavirus\&Itemid=812.

World Health Organization. (2021a). WHO Coronavirus Disease (COVID-19) Dashboard. https://covid19.who.int/.

World Health Organization. (2021b). Coronavirus disease (COVID-19): Vaccines. https://www.who.int/news-room/q-a-detail/coronavirus-disease-(covid-19)vaccines?adgroupsurvey $=\{$ adgroupsurvey $\} \&$ gclid=Cj0KCQiA0-6ABhDMARIsAFVdQv_3iFN8oJGQSguzeE3njUMY9J8svN DMtvvnUuoFWt76 CXIdS36n 8Ks aA gS BEALw_wcB.

World Health Organization. (2021c). COVID-19 Vaccines. https://www.who.int/emergencies/diseases/novel-coronavirus-2019/covid-19-vaccines.

Velavan, T. P., \& Meyer, C. G. (2020). A epidemia de COVID-19. Medicina tropical e saúde internacional: TM \& IH, 25 (3), $278-280$. https://doi.org/10.1111/tmi.13383 